\title{
Combining transcutaneous electrical nerve stimulation with therapeutic exercise to reduce pain in an elderly population: a pilot study
}

Running head: Electrical nerve stimulation and exercise to reduce pain

Article category: Research paper

Simon Vaillancourt ${ }^{1} \neq$, Alexia Coulombe-Lévêque ${ }^{1,2} \neq$, Julie Fradette ${ }^{1}$, Stéphanie Martel ${ }^{1}$, Wafaa Naour ${ }^{1}$, Rubens Da Silva ${ }^{3}$, Guillaume Léonard ${ }^{1,2}$

${ }^{1}$ School of Rehabilitation, Université de Sherbrooke, Sherbrooke, Quebec, Canada

${ }^{2}$ Research Centre on Aging, Centre intégré universitaire de santé et de services sociaux de l'Estrie - Centre hospitalier universitaire de Sherbrooke (CIUSSS de l'Estrie-CHUS), Sherbrooke, Quebec, Canada

${ }^{3}$ Health Sciences Department, Université du Québec à Chicoutimi, Chicoutimi, Quebec, Canada

₹ Co-first authors

Corresponding author: $\operatorname{Pr}$ G. Léonard. *0000-0002-3625-3072* Research Center on Aging, CIUSSS de l'Estrie-CHUS, 1036 rue Belvédère Sud, Sherbrooke (Québec), J1H 4C4, Canada, guillaume.leonard2@usherbrooke.ca

\section{Declaration of Interest}

The authors report no declarations of interest. This study was supported by internal funding from the School of Rehabilitation (Université de Sherbrooke). Alexia Coulombe-Lévêque was supported by a summer scholarship from the Réseau québécois de recherche en adaptationréadaptation. Guillaume Léonard is supported by the Fonds de la recherche en santé du québec.

\section{Author contributions}


GL, SV, JF, SM and WN designed the study; SV, JF, SM and WN performed the experiment and collected the data; ACL analysed the data and wrote the manuscript, with the help of SV, JF, SM and WN, RDS and GL. RDS and GL supervised the project.

Word Count: 4340 words (excluding abstract, references, and tables) 


\section{ABSTRACT}

Purpose: Chronic pain is a highly prevalent and debilitating condition, and there is a pressing need to find safe, effective and affordable treatments to tackle this public health issue. This pilot study aimed to assess whether therapeutic exercises supplemented by transcutaneous electrical nerve stimulation induces a greater hypoalgesic effect than therapeutic exercises supplemented by sham transcutaneous electrical nerve stimulation, in an elderly population suffering from chronic pain.

Materials and methods: Eighteen elderly participants suffering from chronic pain completed a therapeutic exercise program consisting of 45-min group sessions administered twice a week for four weeks. Half of the participants received real transcutaneous electrical nerve stimulation during the exercise sessions, while the others received sham transcutaneous electrical nerve stimulation. Participants completed pain questionnaires (McGill Pain Questionnaire, Brief Pain Inventory, Beck Depression Index) before and after the intervention, and recorded their pain levels on an 11-point numerical rating scale before and after each session (Clinical Trial.Gov ID : NCT02445677).

Results and conclusion: Our results suggest that supplementing exercise sessions with transcutaneous electrical nerve stimulation does not improve the long-term outcomes of elderly patients suffering from chronic pain, but does induce short-term hypoalgesia during exercise sessions. Our study also offers valuable guidelines for the implementation of a future and adequately powered study looking at this research question.

Keywords: Transcutaneous electrical nerve stimulation; Exercise; Chronic Pain; Pain; Aged. 


\section{Introduction}

The proportion of people aged 65 and older is rising in Canada, from $8 \%$ in 1960 to $16 \%$ in 2015. It is predicted to continue increasing, reaching up to $25 \%$ of the Canadian population by 2035 [1]. Such aging of the population is of great significance from a public health standpoint, as many health conditions - including chronic pain - become significantly more prevalent with age $[2,3]$. Chronic pain is made particularly difficult to treat in elderly patients by the elevated rates of comorbidities, and by the presence of polymedication and its oftenincapacitating side-effects $[4,5]$. It is essential that effective, low-cost treatment protocols be developed for the elderly population suffering from chronic pain.

Therapeutic exercises and transcutaneous electrical nerve stimulation (TENS), two modalities frequently used by physical therapists, that have been proposed as treatment options for chronic pain [6-11]. TENS, perhaps the lesser known of these two interventions, consists in applying electrical stimulation through skin electrodes to depolarize peripheral nerve fibers and induce hypoalgesia [12]. Multiple studies have demonstrated the hypoalgesic effects of TENS in patients suffering from various conditions, such as migraines, knee osteoarthritis, multiple sclerosis, and pelvic pain [13-16]. There is evidence to show that age can influence patients' response to TENS. For instance, a recent study evaluating the effects of TENS in different age groups (young adults, middle-aged adults, older adults) has shown that while all participants experienced hypoalgesia and decreased pain sensitivity following TENS, a greater stimulation intensity was required to induce hypoalgesia in elderly participants [17]. Unfortunately, because this study did not include a control condition, we cannot determine whether the observed hypoalgesia resulted from the TENS or from a placebo effect. Moreover, while elderly participants seem more responsive to the placebo effect [18], 
at least one study suggests they may also be less susceptible to TENS-induced hypoalgesia than younger subjects [19].

Treatment approaches that include both TENS and therapeutic exercises have been studied [20-24], but only on adult participants, and never with the two modalities superimposed on each other. The study by Hawamdeh et al. (2014) [23], which comes closest to a concomitant application (TENS and exercises were applied sequentially: 15 minutes of TENS immediately followed by 15 minutes of exercise), indeed seems to show better outcomes in the real TENS group compared to the sham TENS group, although the scarcity of quantifiable data reported makes this study difficult to interpret. Considering these results, as well as the varying response to TENS observed across different age groups and the special challenges faced by an aged population mentioned previously, there is a need to evaluate the pain-relief effects of joint therapeutic exercises + TENS program specifically in elderly patients.

Accordingly, we designed a protocol to study whether therapeutic exercises supplemented by TENS induce a greater hypoalgesic effect than therapeutic exercises supplemented by sham TENS, in an elderly population suffering from chronic pain. The objectives of the present pilot study were to estimate the effectiveness and effect size of our intervention, and to assess the feasibility of our protocol for the implementation of a future, adequately powered randomized controlled trial looking at the combined effect of TENS and therapeutic exercises in elderly individuals suffering from chronic pain.

\section{Material and methods}

\section{Study Design}

We conducted a single-blind randomized controlled trial to test our hypothesis. Data was collected over two intervention periods, the first one in February 2017 and the second in July 
2017. The intervention protocols were carried out over 4 weeks, and consisted of eight 45 minute-long group therapeutic exercise sessions administered twice a week. Participants were randomly assigned to the experimental and control groups using a random number table designed by the statistician of the Research Center on Aging, using Minitab Statistical Software (version 15.0; State College, PA). The principal investigator assigned the participants to the two groups. The participants and the person assessing the outcomes were blinded to the interventions during the entire study. The experimental group received real TENS stimulation during therapeutic exercise sessions, whereas the control group received sham TENS stimulation during those same sessions. Our protocol satisfied the ethical standards of the local institutional research committee (Centre intégré universitaire de santé et de services sociaux de l'Estrie - Centre hospitalier universitaire de Sherbrooke - \#2017-671Institut universitaire de gériatrie de Sherbrooke), in accordance with the Declaration of Helsinki of 1964 and its later amendments. All participants signed an informed consent form, approved by the research committee, before taking part in the study. This clinical trial was registered in Clinical Trial.Gov (ID : NCT02445677) before enrolment of the first participant.

\section{Participants}

To be included in our study, participants were required to be aged 65 or older, speak French, and suffer from chronic (> 6 months) pain. We excluded participants with unstable analgesic medication, a diagnosis of cancer, cognitive deficits, prior experience with TENS, or for whom TENS was contraindicated [see 12]. In order to optimize the efficacy of the TENS, we asked participants to refrain from consuming caffeine during the 6 hours preceding each treatment session [22]. The study (recruitment and intervention) was conducted in two retirement residences in the Sherbrooke area: Chartwell Villa de l'Estrie (February 2017) and Chartwell Seigneuries du Carrefour (July 2017). 


\section{Therapeutic Exercise Program}

The therapeutic exercise program consisted of group training sessions taking place twice a week over a period of four weeks, under the supervision of fourth-year physiotherapy students (with ample knowledge to adequately oversee the exercises and handle TENS). Sessions lasted 45 minutes and were comprised of a general cardiovascular warm-up (15 mins) followed by strengthening exercises targeting major muscle groups in the four limbs and using elastic bands for resistance (20 mins), and ending with stretching and relaxation (10 mins) (see Supplementary Table S1 for a more detailed breakdown of the sessions). Sessions all took place in the early afternoon and were spread throughout the week to allow for appropriate (>36h) recovery.

\section{TENS}

This study used the Biomed 2000XL 2-channel TENS unit manufactured by Vitality Medical, with carbon rubber electrodes. The electrodes were applied on each participant's most painful region, as identified at the beginning of the first session. The TENS parameters were set as per conventional mode recommendations $(100 \mathrm{~Hz}, 60 \mu \mathrm{s})$, and the intensity was individually adjusted such that the stimulation was intense but not painful [12]. For participants in the experimental group, TENS intensity remained constant throughout the entire therapeutic exercise session. For participants in the control group, TENS intensity was maintained for 15 seconds before being gradually reduced; the TENS machines were then turned off for the entire duration of the exercise session, unbeknownst to the participants. To ensure that participants remained blinded to the experimental conditions, they were systematically told that TENS is perceived differently by every individual and that it is normal for some people to feel it more or less than others. 


\section{Outcome Measures}

All outcome measures were selected as per recommendations from the Initiative on Methods, Measurement, and Pain Assessment in Clinical Trials [25,26]. We used the Short-Form McGill Pain Questionnaire to assess the sensory and affective quality of pain in our subjects. This tool allows participants to rate the extent to which given descriptors (ex. "stabbing", "dull”, "suffocating", etc.) apply to their pain, such that a higher score corresponds to greater pain, out of a maximum score of 45 [27]. This tool shows adequate content validity, sensitivity to change $(>0.8)$, and test-retest reliability $(0.75-0.93)$, and is widely used in pain research $[25-28]$.

We used the Brief Pain Inventory to assess the severity of pain and the interference of pain with physical functioning. This tool provides two outcome measures (severity of pain [questions 3-6], out of 40; and interference of pain with physical functioning [question 9 a-g], out of 70), and has been shown to have satisfactory test-retest reliability (0.83-0.98) and validity (Cronbach's alpha $=0.77-0.91)[25,26,29]$.

We used the Beck Depression Inventory to assess the impact of pain on emotional functioning. This tool consists of 21 questions, each with at least four possible answers ranging in intensity (0-3, for a maximum score of 63$)$. The validity of this questionnaire depends on depressive symptoms having been present for at least 3 weeks [39]. This tool shows adequate test-retest reliability (0.6-0.9) and good sensitivity (89\%) and specificity (90\%) when scores are greater than $21[25,26,31]$.

We used a 0-10 numerical rating scale to assess pain intensity (0: no pain; 10: worst pain imaginable) before and after each training session. We selected this scale because it is easier to understand than the visual analog scale for an elderly population [25]. It has relatively 
high reliability [32], and has been demonstrated to be adequate when studying an elderly population suffering from persistent pain $[25,26,33,34]$.

Pain intensity was assessed before and after each of the 8 training sessions, for a total of 16 data points per participants. The three questionnaires were administered twice: once during the week preceding the first training session (T0), and a second time a week after the final (8th) training session (T1).

\section{Statistical analysis}

Between-group comparisons were made using Student's t-tests. Demographic characteristics were compared to make sure that the two groups were similar. Questionnaire scores (obtained at $\mathrm{T} 0$ and $\mathrm{T} 1$ ) were compared to rule out an initial difference between the two groups and to test for between-group difference after the intervention. The magnitude of improvement (i.e. $\Delta$, the change in questionnaire scores between $\mathrm{T} 0$ and $\mathrm{T} 1$ calculated as $\mathrm{T} 0-\mathrm{T} 1$ ) was also compared between the two groups for all questionnaires, to determine whether the experimental group had improved to a greater extent than the control group. Finally, withingroup analysis (paired-sample t-tests) were used to assess whether our intervention had induced a statistically significant change in either group. Because of the small sample size $(n=18)$ and the results from the Kolmogorov-Smirnov tests suggesting the presence of nonnormally distributed data for a small number of variables, non-parametric tests were additionally used for between- (Mann-Whitney $\mathrm{U}$ test) and within-group (Wilcoxon signedrank test) comparisons. Results confirmed no difference between parametric and nonparametric approaches; therefore, only parametric statistics are reported.

Independent and paired-sample t-tests as well as non-parametric equivalents were performed using SPSS (version 19.0 for Windows, Chicago, IL, USA). In an effort to increase reproducibility and reduce the rate of false positives in the literature, and to promote scientific 
rigor within the field, we embrace the changes proposed by Benjamin et al. [35], wherein the threshold for statistical significance is lowered to $\mathrm{p}<0.005$ and $\mathrm{p}$-values ranging between 0.05 and 0.005 are instead considered "suggestive".

The absolute and relative changes in pain intensity were calculated for each participant over each session. Given the large proportion of missing pain scores (see the Results and Discussion sections), we did not attempt to establish statistical significance for this outcome measure, instead keeping only to a descriptive analysis; as per recommendations from the Initiative on Methods, Measurement, and Pain Assessment in Clinical Trials, we report absolute changes in pain intensity (minimal clinical change: $\Delta \geq 2$ ), as well as the percentage of patients obtaining reduction in pain intensity of at least $30 \%$ and $50 \%$ from baseline [25].

\section{Results}

\section{Participants}

In total, 21 participants (aged $83 \pm 4$ years old) met our criteria, but three of them dropped out before the beginning of the first session (due to lack of interest or scheduling conflicts), such that 18 participants gave their informed consent and took part in the experiment. Our sample consisted of only women, as no men volunteered to participate in our study. Eleven participants were recruited from Villa de l'Estrie, and seven from Les Seigneuries du Carrefour. Six participants were suffering from axial pain, five from lower limb pain, and seven from upper limb pain. Participants had been suffering from chronic pain for an average of 22 years.

Our protocol planned for eight training sessions delivered twice a week for 4 weeks. Unfortunately, a flu epidemic at Villa de l'Estrie made it impossible to deliver the last training session; those participants thus attended only up to 7 sessions each. All sessions successfully took place at Seigneuries du Carrefour. Attendance to the training sessions was similar across 
the experimental and control groups (average participation: $5 \pm 2$ sessions). Three participants from Villa de 1'Estrie (one from the experimental group, two from the control group) discontinued their participation in the study during the intervention period (one participant caught the zona virus; another lost interest in the study; the last had too many other medical appointments to attend). In order to complete data analysis, we accounted for the three drop outs as follows: for the questionnaires, we set missing $\mathrm{T} 1$ scores equal to their respective $\mathrm{T} 0$ scores; for the pain scores (recorded at the beginning and end of each session), we retained scores for the attended sessions, such that the drop out participants were no different from other participants with imperfect attendance.

\section{Outcome measures}

\section{Questionnaires}

We found that it was necessary for a team member to assist our participants while they were filling in the questionnaires, as participants were often unsure how to answer questions. Results from the three questionnaires can be found in Table 1.

[Insert Table 1 here]

Independent t-tests showed no statistically significant difference in post-treatment (T1) scores between groups and paired-sample t-tests for dependent samples showed no statistically significant improvement on any questionnaires (all p-values $>.05$; see Table 1). The only statistically suggestive effect was observed on McGill Pain Questionnaire scores: the experimental group showed a small decrease in scores, while the control group showed a smaller still increase in scores, such that the evolution appears different between the two 
groups $(\mathrm{p}=0.04)$; however, this evolution was not clinically significant in either group (minimal clinical difference $\geq 5$ ).

\section{Numerical pain rating scale}

We measured pain intensity using the numerical rating scale at the beginning and end of each training session to determine whether TENS potentiated the short-term hypoalgesic effects of exercise. In total, we aimed to collect $144(18$ participants $* 8$ sessions $=144)$ pairs of prepost pain scores; however, because of poor attendance and drop outs, we were only able to collect 98 data pairs (52 in the experimental group, and 46 in the control group - 32\% missing data overall). Moreover, despite our inclusion criteria requiring that all our participants suffer from chronic pain, we had an unfortunately large number of initial pain scores of 0 throughout the intervention (15 in the experimental group, and 5 in the control group, for a total of 20 out of 98 data pairs). An absence of pain in our patients is desirable in the grand scheme of things, but it obviously generates a substantial floor effect for our intervention. We therefore elected to exclude those 20 data pairs with an initial score of 0 . While this might have jeopardized our ability to draw statistically significant conclusions, our $32 \%$ missing data had already done away with that option, and inclusion of these data would have artificially decreased the effect of our intervention and biased our group comparisons by virtue of their asymmetrical distribution. For the sake of transparency, we have included in the supplementary materials a replication of our analysis which does not exclude those 20 data pairs - as expected, results obtained this way follow the exact same trend as the results presented in the main text, albeit with smaller effects.

[Insert Table 2 here] 
The average hypoalgesia induced in the two groups during each session is shown in Table 2, and was calculated as an average of the absolute (and relative) hypoalgesia induced in each participant, as per their pre- and post-session pain scores. The number of data points making up each average (i.e. the number of participants present for the session and with a pre-session score greater than 0$)$ is reported. Notably, while no session induced significant $(\geq 2)$ pain relief in the control group, 5 out of 8 training sessions induced clinically significant pain relief in the experimental group. With a significance threshold of $\geq 30 \%$, this figure increases to 6 sessions.

[Insert Table 3 here]

The average hypoalgesia induced in each participant similarly also favors the experimental group over the control group (Table 3). These scores were obtained by averaging the absolute (and relative) hypoalgesia induced in each participant as per their pre- and post-session pain scores. The number of data points making up each average (i.e. the number of sessions attended and with a pre-session score greater than 0 ) is reported. Six experimental participants experienced clinically significant hypoalgesia ( $\geq 2$ and $\geq 30 \%$ ) on average when they attended the sessions, compared to only two control participants.

[Insert Table 4 here]

The average hypoalgesia induced across all sessions and all participants (calculated as the average of all data points) was clinically significant in the experimental group, but not in the 
control group (Table 4). The proportion of sessions during which significant hypoalgesia was induced (calculated as the number of instances for which the delta in pain scores met the threshold, divided by the total number of delta pain scores recorded) is also reported for both groups with respect to the various thresholds.

\section{Discussion}

This pilot study assessed the effect of an exercise program combined with conventional TENS, compared to an exercise program combined with sham TENS, on chronic pain in an elderly population.

\section{Effectiveness of the intervention}

Results obtained from the three questionnaires at baseline and post-intervention show no difference between the two groups, except for a statistically suggestive difference in McGill Pain Questionnaire score changes $(\Delta)$ between the experimental and control groups postintervention, resulting from a non-clinically significant improvement (the McGill Pain Questionnaire threshold for clinical significance is $\Delta \geq 5$, out of a maximum score of 45 ; see Strand et al. [28]). Our results therefore suggest that combining TENS with a training program does not improve the overall treatment outcome in terms of quality of pain, physical functioning, and emotional functioning in elderly patients suffering from chronic pain.

\section{Pain intensity}

While the results reported in Table 2 seem to indicate that the hypoalgesia induced during each session was greater in the experimental group than in the control group, it is important to keep in mind that the number of data points making up each average varies between groups and between sessions (as a function of attendance and the data excluded 
because of an initial pain score of 0 ). Because of this, session averages may be overly influenced by outliers, should these outliers occur in low-attendance sessions. For instance, the average hypoalgesia observed in the experimental group during session 8 seems remarkable - but this value only comprises data from two participants, one of which reported an hypoalgesia of 8 . As such, this figure can be rather misleading if it is not interpreted attentively. Nonetheless, while the size of the effect is difficult to estimate accurately at a glance, a clear trend favoring the experimental group transpires from the data.

The average hypoalgesia induced in each participant throughout the training sessions (Table 3) perhaps constitutes a more valid and more easily interpretable outcome measure, because it is not biased by attendance; each result reflects the average hypoalgesia experienced by a given participant, regardless of the participant's attendance - and, more importantly, regardless of the overall group attendance. Similarly, while the average hypoalgesia induced in both groups and the proportion of hypoalgesia-inducing sessions in both groups (Table 4) were obtained from a relatively small data set and might accordingly be biased by random noise, these results are not at risk of being systematically biased by the attendance rate.

Considering the limitations and possible biases listed above, we recognize that any one of our results, taken individually, does not offer robust evidence supporting the addition of TENS to an exercise program. We should also reiterate that statistical significance was not established, and that our analysis is purely descriptive. However, the fact that our results consistently and unequivocally converge in favor of the experimental group, regardless of how the data is analyzed, lends a certain degree of credibility to our conclusion that exercise sessions supplemented with TENS probably result in short-term hypoalgesia, unlike exercise sessions alone. 


\section{Limitations and recommendations for future trials}

\section{Sample}

The first and most obvious limitation in our study is the low attendance rate we experienced. A follow-up study should consider adopting strategies to increase attendance (e.g. phone reminders), while also accounting for the expected low attendance when determining the study sample size. The site of musculoskeletal injury/pain may also correlate with the success of the intervention [36] - a variable that has not yet been studied, to the best of our knowledge. A larger sample would be needed in order to investigate this possible effect.

Given that our sample consists only of women, we cannot generalize our results to male patients. A larger-scale study would do well to attempt to recruit a significant proportion of male participants, to increase the external validity of the study. Sex (and/or gender) is an important factor to consider, seeing as some studies have shown that women perceive pain differently than men. For instance, women seem to have a lower pain threshold than men [37], and have been said to preferentially recruit "emotional brain circuits" when subjected to pain [38]. Unfortunately, men suffering from chronic pain have a lower tendency than women to seek treatment [39], which makes the gender distribution of our sample unsurprising, if undesirable.

Some of our results might also have been biased by our participants exhibiting relatively mild symptoms. For instance, the low McGill Pain Questionnaire scores obtained at baseline could have potentially led to a floor effect, artificially limiting the effect size of our intervention. Similarly, the absence of pain reported relatively frequently by our participants at the beginning of training sessions considerably limited our ability to generate hypoalgesia in those patients. In order to avoid these problems, a follow-up study should 
consider narrowing the inclusion criteria, for example by requiring that participants suffer from constant pain.

\section{Instruments}

The tools we had selected to assess outcome (pain scale and questionnaires) proved surprisingly difficult to understand for our patients. This was unexpected, as they are all wellvalidated and extremely common in pain research. Eventually, all participants understood how to answer the various questionnaires, but sometimes only after several rounds of explanations. Therefore, while these tools are designed to be self-administered, our experience (see also Harvey et al. [40]) shows that the presence of a research team member is necessary during completion of the questionnaires, to ensure that the results obtained are valid, at least for elderly study participants.

Another limitation in our study relates to TENS parameters. In order to yield optimal results, conventional TENS intensity should be adjusted throughout a training session, such that the stimulation is always perceived as being intense but non-painful (as the nervous system habituates to the stimulation, patients feel the TENS as being less and less intense, which must be corrected for by gradually increasing the intensity, often multiple times within a session; see Bélanger [12]; Moran et al. [41]; and Pantaleao et al. [42]. However, in order to keep our participants blinded to their group allocation, we chose not to increase TENS intensity throughout the training session. This might have resulted in suboptimal TENS parameters, which could have translated in decreased treatment effects [42]. On the other hand, a recent study conducted on patients suffering from clinical pain (as opposed to the experimental pain induced by Pantaleao et al. [42]) has shown that adjusting the TENS intensity throughout a session had no effect on the hypoalgesia induced [43]. In any case, it would be possible for a future study to avoid this limitation altogether by telling participants 
they would either receive 'conventional' (experimental group) or 'microcurrent' (control group) TENS. Therapists could then adjust stimulation intensity in both groups throughout training sessions, informing experimental participants that the sensation should be intense but not painful, while informing control participants that the stimulation should remain 'undetectable'.

Finally, seeing as no long-term follow-up assessment took place, we cannot determine whether our intervention had any longer lasting effects. As such, it would be worthwhile in a future study to include follow-up measures, for example 4 weeks and 8 weeks after the end of the training program

\section{Conclusion}

This pilot study offers valuable guidelines for the implementation of a future study on the effect of TENS combined with therapeutic exercises. Our strongest recommendation would be to select participants more sharply: a larger, gender-balanced sample will generate more reliable results that can be generalized to a larger population; and a more symptomatic sample will generate results that are less likely to be affected by a potential floor effect.

Our results suggest that supplementing exercise sessions with TENS does not improve the overall outcome of elderly patients suffering from chronic pain, but does result in shortterm hypoalgesia throughout each training session. As such, it is plausible that this treatment approach might indirectly benefit a patient population, for example by improving tolerance to effort, or by decreasing maladaptive pain-related cognitions such as catastrophisation and kinesiophobia. Obviously, these are but speculations, and require much further investigation to be considered. Our results, as they stand, are promising enough to justify such investigation be undertaken. 


\section{Acknowledgements}

The authors would like to thank Mr. Daniel Mireault, Ms. Émilie Lanoix and Véronique Poirier for their help with data collection; Ms. Judith Paquin-Veillette for her contribution to the writing of the manuscript; and finally, the Chartwell residences and all the subjects who participated in this project 


\section{References}

[1] Statistics Canada [internet]. Estimations de la population du Canada: âge et sexe. [cited 2015 sept 29]. Available from: https:/www.statcan.gc.ca/dailyquotidien/150929/dq150929b-fra.htm

[2] Elliott AM, Smith BH, Penny KI, et al. The epidemiology of chronic pain in the community. Lancet. 1999;354(9186):1248-1252.

[3] Ramage-Morin PL. Medication use among senior Canadians. Health Rep. 2009;20(1):37-44.

[4] Molton IR, Terrill AL. Overview of persistent pain in older adults. Am Psychol. 2014;69(2):197-207.

[5] Rao A, Cohen HJ. Symptom management in the elderly cancer patient: fatigue, pain, and depression. J Natl Cancer Inst Monogr. 2004;32:150-157.

[6] Geneen LJ, Moore RA, Clarke C, et al. Physical activity and exercise for chronic pain in adults: an overview of Cochrane Reviews. Cochrane Database Syst Rev. 2017;14(1):CD011279.

[7] Jauregui JJ, Cherian JJ, Gwam CU, et al. A meta-analysis of transcutaneous electrical nerve stimulation for chronic low back pain. Surg Technol Int. 2016;28:296-302.

[8] Sluka KA, Bjordal JM, Marchand S, et al. What makes transcutaneous electrical nerve stimulation work? Making sense of the mixed results in the clinical literature. Phys Ther. 2013;93(10):1397-1402.

[9] Rutjes AW, Nuesch E, Sterchi R, et al. Transcutaneous electrostimulation for osteoarthritis of the knee. Cochrane Database Syst Rev. 2009;7(4):CD002823. 
[10] Levy SS, Macera CA, Hootman JM, et al. Evaluation of a multi-component group exercise program for adults with arthritis: Fitness and Exercise for People with Arthritis (FEPA). Disabil Health J. 2012;5(4):305-311.

[11] Tse MM, Vong SK, Ho SS. The effectiveness of an integrated pain management program for older persons and staff in nursing homes. Arch Gerontol Geriatr. 2012;54(2):e203-212.

[12] Bélanger AY. Therapeutic electrophysical agents: evidence behind practice. 3rd ed. Philadelphia (PA): Lippincott Williams \& Wilkins; 2015.

[13] Chen LX, Zhou ZR, Li YL, et al. Transcutaneous electrical nerve stimulation in patients with knee osteoarthritis: evidence from randomized-controlled trials. Clin J Pain. 2016;32(2):146-154.

[14] Sawant A, Dadurka K, Overend T, et al. Systematic review of efficacy of TENS for management of central pain in people with multiple sclerosis. Mult Scler Relat Dis. 2015;4(3):219-227.

[15] Sharma N, Rekha K, Srinivasan JK. Efficacy of transcutaneous electrical nerve stimulation in the treatment of chronic pelvic pain. J Midlife Health. 2017;8(1):3639.

[16] Tao H, Wang T, Dong X, et al. Effectiveness of transcutaneous electrical nerve stimulation for the treatment of migraine: a meta-analysis of randomized controlled trials. Journal Headache Pain. 2018;19(1):42.

[17] Simon CB, Riley JL 3rd, Fillingim RB, et al. Age group comparisons of TENS response among individuals with chronic axial low back pain. J Pain. 2015;16(12):1268-1279. 
[18] Daguet I, Bergeron-Vezina K, Harvey MP, et al. Transcutaneous electrical nerve stimulation and placebo analgesia: is the effect the same for young and older individuals? Clin Interv Aging. 2018;13:335-342.

[19] Bergeron-Vezina K, Corriveau H, Martel M, et al. High- and low-frequency transcutaneous electrical nerve stimulation does not reduce experimental pain in elderly individuals. Pain. 2015;156(10):2093-2099.

[20] Dissanayaka TD, Pallegama RW, Suraweera HJ, et al. Comparison of the effectiveness of transcutaneous electrical nerve stimulation and interferential therapy on the upper trapezius in myofascial pain syndrome: a randomized controlled study. Am J Phys Med Rehab. 2016;95(9):663-672.

[21] Mutlu B, Paker N, Bugdayci D, et al. Efficacy of supervised exercise combined with transcutaneous electrical nerve stimulation in women with fibromyalgia: a prospective controlled study. Rheumatol Int. 2013;33(3):649-655.

[22] Marchand S. Le phénomène de la douleur. Montréal (QC): Les Éditions de la Chenelière inc; 2009.

[23] Hawamdeh M, Al Malty AAM, Tariah HA, Omar MH et al. Comparison between the Effectiveness of using Transcutaneous Electrical Nerve Stimulation (TENS) with Back Strengthening Exercises and the use of Back Strengthening Exercises only on Patients with Chronic Low Back Pain: a Pilot Crossover Double Blind Study. Indian J Physiother Occup Ther. 2015;9(2) : 210-2014.

[24] Deyo RA, Walsh NE, Martin DC et al. A controlled trial of transcutaneous electrical nerve stimulation (TENS) and exercise for chronic low back pain. N Engl J Med. $1990 ; 322(23): 1627-1634$. 
[25] Dworkin RH, Turk DC, Farrar JT, et al. Core outcome measures for chronic pain clinical trials: IMMPACT recommendations. Pain. 2005;113(1-2):9-19.

[26] IMMPACT [internet]. Initiative on methods, measurement, and pain assessment in clinical trials. Available from: http://www.immpact.org/

[27] Melzack R. The short-form McGill Pain Questionnaire. Pain. 1987;30(2):191-197.

[28] Strand LI, Ljunggren AE, Bogen B, et al. The Short-Form McGill Pain Questionnaire as an outcome measure: test-retest reliability and responsiveness to change. Eur $\mathbf{J}$ Pain. 2008;12(7):917-925.

[29] Cleeland CS, Ryan KM. Pain assessment: global use of the Brief Pain Inventory. Ann Acad Med Singapore. 1994;23(2):129-138.

[30] Beck AT, Steer RA, Brown GK. Manual for the Beck depression inventory-II. San Antonio (TX): Psychological Corp; 1996.

[31] Harris CA, D'Eon JL. Psychometric properties of the Beck Depression Inventorysecond edition (BDI-II) in individuals with chronic pain. Pain. 2008;137(3):609-622.

[32] Boonstra AM, Schiphorst Preuper HR, Reneman MF, et al. Reliability and validity of the visual analogue scale for disability in patients with chronic musculoskeletal pain. Int J Rehabil Res. 2008;31(2):165-169.

[33] Wood BM, Nicholas MK, Blyth F, et al. Assessing pain in older people with persistent pain: the NRS is valid but only provides part of the picture. J Pain. 2010;11(12):1259-1266.

[34] Kimura T, Hayashida K, Araki Y, et al. How valid is the self-administered visual analogue scale for assessing the health status of elderly people? Hiroshima J Med Sci. 2008;57(3-4):85-92. 
[35] Benjamin DJ, Berger JO, Johannesson M, et al. Redefine statistical significance. Nat Hum Behav. 2018;2(1):6-10.

[36] Johnson M, Martinson M. Efficacy of electrical nerve stimulation for chronic musculoskeletal pain: a meta-analysis of randomized controlled trials. Pain. 2007;130(1-2):157-165.

[37] Hashmi JA, Davis KD. Deconstructing sex differences in pain sensitivity. Pain. 2014;155(1):10-13.

[38] Girard-Tremblay L, Auclair V, Daigle K, et al. Sex differences in the neural representation of pain unpleasantness. J Pain. 2014;15(8):867-877.

[39] Institut de la statistique du Québec [internet]. Aubin J, Traoré I. La consultation de professionnels de la santé par les Québécois: comparaisons temporelles et provinciales. [cited 2009 Mar 1] Available from: http://www.stat.gouv.qc.ca/statistiques/sante/bulletins/zoom-sante-200703.pdf

[40] Harvey MP, Lorrain D, Martel M, et al. Can we improve pain and sleep in elderly individuals with transcranial direct current stimulation? Results from a randomized controlled pilot study. Clin Interv Aging. 2017;12:937-947.

[41] Moran F, Leonard T, Hawthorne S, et al. Hypoalgesia in response to transcutaneous electrical nerve stimulation (TENS) depends on stimulation intensity. J Pain. 2011;12(8):929-935.

[42] Pantaleao MA, Laurino MF, Gallego NL, et al. Adjusting pulse amplitude during TENS application produces greater hypoalgesia. J Pain. 2011;12(5):581-590.

[43] Bergeron-Vezina K, Filion C, Couture C, et al. Adjusting pulse amplitude during transcutaneous electrical nerve stimulation does not provide greater hypoalgesia. $\mathrm{J}$ Altern Complement Med. 2018;24(3):262-267. 
Table 1. Pain Questionnaires

Table 2. Average Hypoalgesia per Session

Table 3. Average Hypoalgesia per Participant

Table 4. Average Hypoalgesia per Participant per Session 
Table 1 - Pain Questionnaires

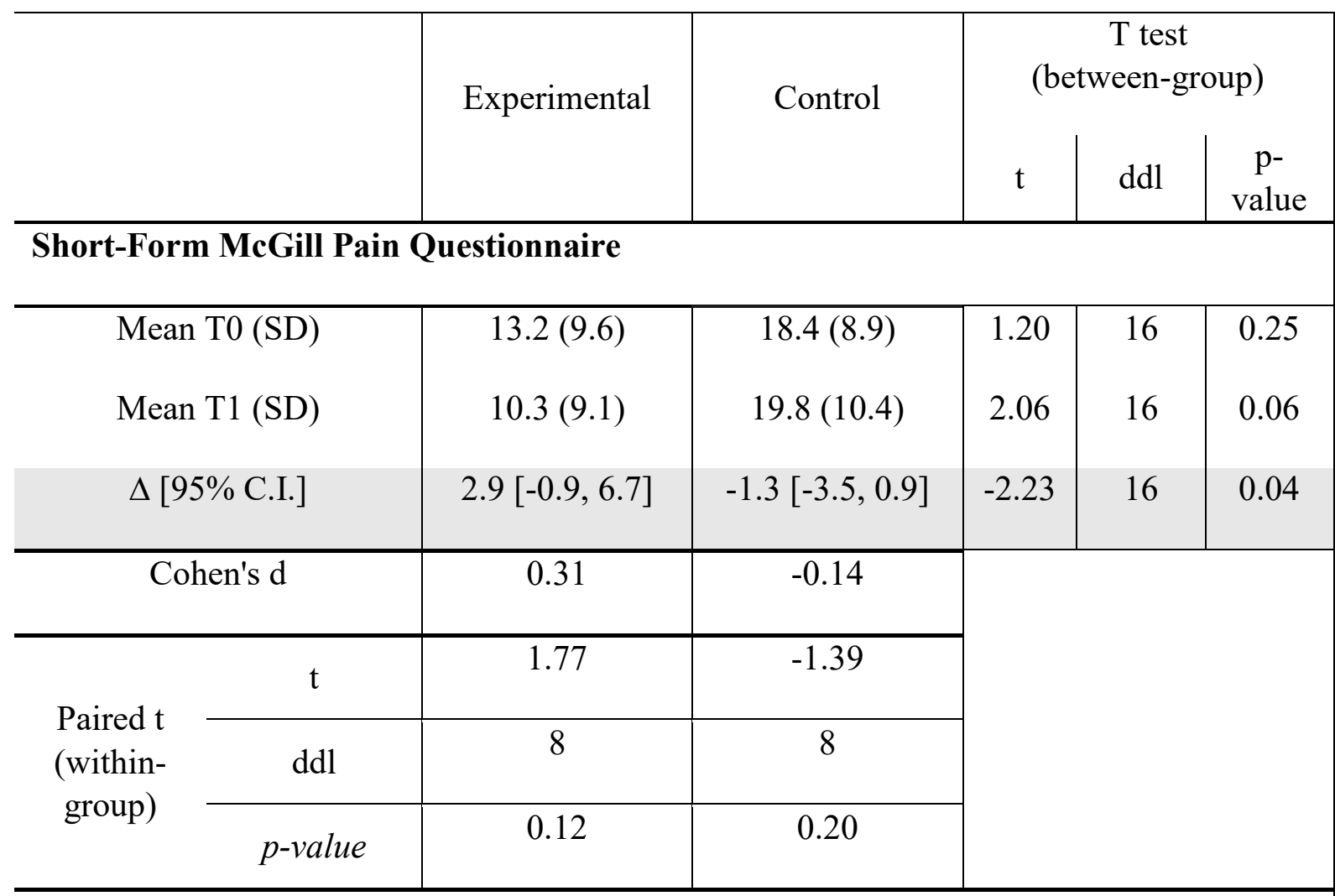

\section{Brief Pain Inventory - severity}

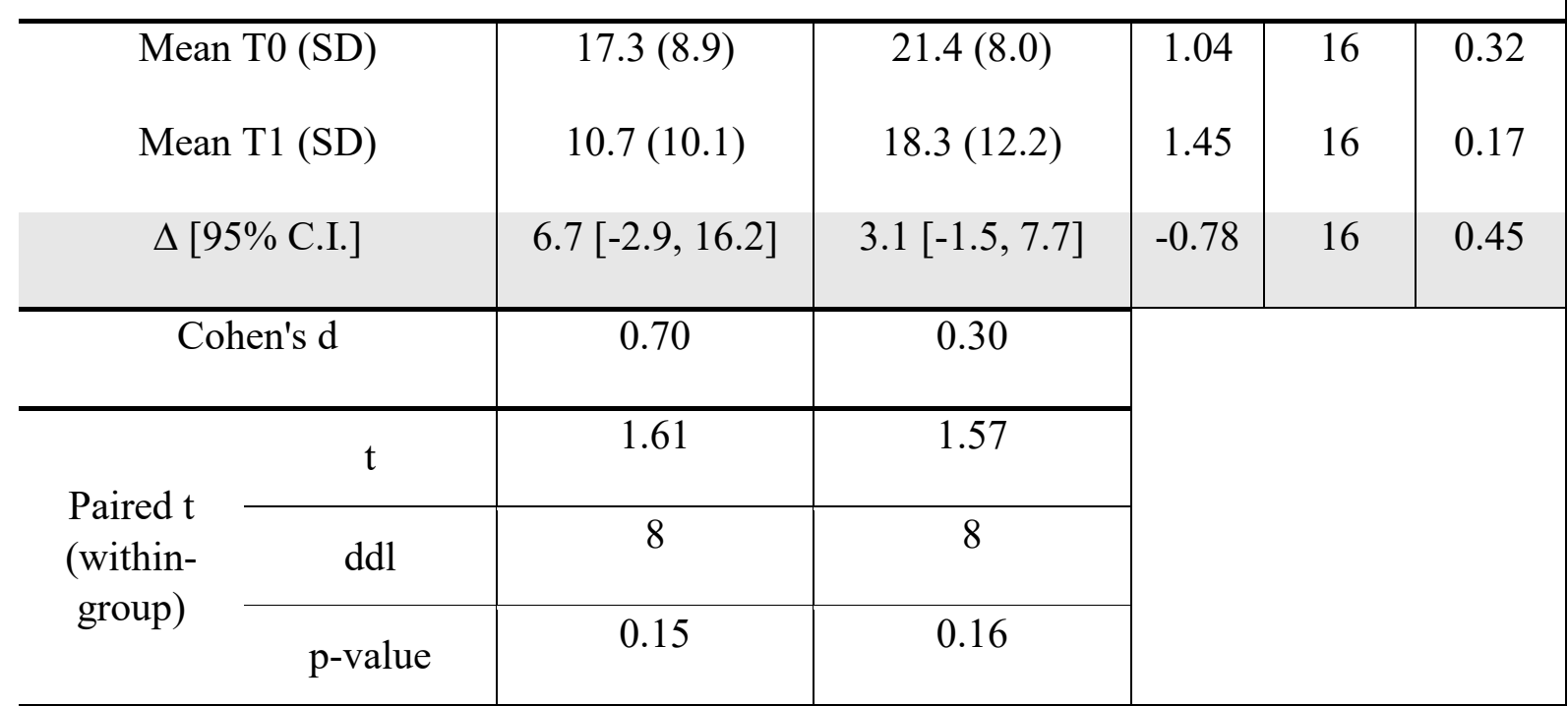

\section{Brief Pain Inventory - function}

\begin{tabular}{|l|c|c|c|c|c|}
\hline Mean T0 (SD) & $17.0(13.9)$ & $24.6(16.8)$ & 1.04 & 16 & 0.31 \\
Mean T1 (SD) & $13.6(13.9)$ & $22.8(19.5)$ & 1.16 & 16 & 0.27
\end{tabular}




\begin{tabular}{|c|c|c|c|c|c|c|}
\hline \multicolumn{2}{|c|}{$\Delta[95 \%$ C.I. $]$} & $3.4[-4.1,11.0]$ & $1.8[-4.7,8.3]$ & -0.39 & 16 & 0.71 \\
\hline \multicolumn{2}{|c|}{ Cohen's d } & 0.25 & 0.10 & & & \\
\hline \multirow{3}{*}{$\begin{array}{l}\text { Paired t } \\
\text { (within- } \\
\text { group) }\end{array}$} & $\mathrm{t}$ & 1.05 & 0.63 & & & \\
\hline & ddl & 8 & 8 & & & \\
\hline & p-value & 0.32 & 0.55 & & & \\
\hline
\end{tabular}

\section{Beck Depression Index}

\begin{tabular}{|c|c|c|c|c|c|c|}
\hline \multicolumn{2}{|c|}{ Mean T0 (SD) } & $9.0(4.5)$ & $10.2(7.9)$ & 0.40 & 16 & 0.69 \\
\hline \multicolumn{2}{|c|}{ Mean T1 (SD) } & $8.9(4.9)$ & $11.0(9.3)$ & 0.61 & 16 & 0.55 \\
\hline \multicolumn{2}{|c|}{$\Delta$ [95\% C.I. $]$} & $0.1[-2.1,2.3]$ & $-0.8[-4.1,2.6]$ & -0.51 & 16 & 0.62 \\
\hline \multicolumn{2}{|c|}{ Cohen's d } & 0.02 & -0.09 & & & \\
\hline \multirow{3}{*}{$\begin{array}{l}\text { Paired t } \\
\text { (within- } \\
\text { group) }\end{array}$} & $\mathrm{t}$ & 0.12 & -0.54 & & & \\
\hline & ddl & 8 & 8 & & & \\
\hline & p-value & 0.91 & 0.61 & & & \\
\hline
\end{tabular}

Table 1. Scores obtained in the experimental and control groups for the Short-Form McGill Pain Questionnaire; the Brief Pain Inventory; and the Beck Depression Index. 
Table 2 - Average Hypoalgesia per Session

Experimental group

\begin{tabular}{l|c|c|c|c|c|c|}
\hline \multirow{2}{*}{ Session } & Session 1 & Session 2 & Session 3 & Session 4 & Session 5 & Session 6 \\
\cline { 1 - 6 } Mean hypoalgesia (absolute) & $\mathbf{2 . 0}$ & 0.3 & $\mathbf{2 . 8}$ & 1.9 & 1.4 & $\mathbf{2 . 2}$ \\
\cline { 1 - 7 } Mean hypoalgesia (relative) & $\mathbf{3 3 \%}$ & $13 \%$ & $\mathbf{4 5 \%}$ & $\mathbf{3 3 \%}$ & $22 \%$ & $\mathbf{3 7 \%}$ \\
\cline { 1 - 7 } & 4 & 3 & 5 & 7 & 5 & 5 \\
\hline
\end{tabular}

Control group

\begin{tabular}{l|c|c|c|c|c|c|}
\hline Session & Session 1 & Session 2 & Session 3 & Session 4 & Session 5 & Session 6 \\
\cline { 1 - 7 } Mean hypoalgesia (absolute) & 0.9 & 0.5 & 0.7 & 0.5 & 1.0 & 0.2 \\
\cline { 1 - 7 } Mean hypoalgesia (relative) & $14 \%$ & $7 \%$ & $11 \%$ & $10 \%$ & $20 \%$ & $3 \%$ \\
\hline
\end{tabular}

Table 2. Absolute and relative hypoalgesia induced in each group over each session $(\mathrm{n}=$ number of participants for each session).

Table 3 - Average Hypoalgesia per Participant

Experimental group

\begin{tabular}{l|ccccccccc}
\hline Subject & E1 & E2 & E3 & E4 & E5 & E6 & E7 & E8 & E9 \\
\hline Mean hypoalgesia (absolute) & 0.0 & n/a & 0.0 & $\mathbf{2 . 0}$ & $\mathbf{3 . 5}$ & $\mathbf{3 . 8}$ & $\mathbf{3 . 0}$ & $\mathbf{4 . 0}$ & $\mathbf{2 . 5}$ \\
\hline Mean hypoalgesia (relative) & $0 \%$ & n/a & $-7 \%$ & $\mathbf{3 6 \%}$ & $\mathbf{4 0 \%}$ & $\mathbf{9 6 \%}$ & $\mathbf{5 6 \%}$ & $\mathbf{1 0 0} \%$ & $\mathbf{4 0 \%}$
\end{tabular}




\begin{tabular}{l|lllllllllllll}
$n=$ & 5 & 0 & 6 & 7 & 4 & 4 & 6 & 1 & 4 \\
\hline
\end{tabular}

\section{Control group}

\begin{tabular}{l|ccccccccc}
\hline Subject & C1 & C2 & C3 & C4 & C5 & C6 & C7 & C8 & C9 \\
\hline Mean hypoalgesia (absolute) & 0.3 & -0.1 & 0.3 & $\mathbf{4 . 0}$ & $\mathbf{2 . 5}$ & 0.5 & 0.3 & 0.3 & 1.4 \\
\hline Mean hypoalgesia (relative) & $8 \%$ & $-4 \%$ & $4 \%$ & $\mathbf{5 0 \%}$ & $\mathbf{1 0 0 \%}$ & $6 \%$ & $4 \%$ & $4 \%$ & $29 \%$ \\
\hline$n=$ & 7 & 7 & 3 & 1 & 2 & 4 & 3 & 7 & 7 \\
\hline
\end{tabular}

Table 3. Average hypoalgesia (absolute and relative) induced in each participant ( $\mathrm{n}=$ number of sessions attended for each participant).

Table 4 - Average Hypoalgesia per Participant per Session

\begin{tabular}{l|c|c|c|c|c|} 
& \multicolumn{2}{|c|}{$\begin{array}{c}\text { Average hypoalgesia per } \\
\text { participant per session }\end{array}$} & \multicolumn{3}{|c|}{ Proportion of hypoalgesia-inducing sessions } \\
\cline { 2 - 6 } & Absolute & Relative & $\begin{array}{c}\Delta \text { pain score } \\
\geq 2\end{array}$ & $\begin{array}{c}\Delta \text { pain score } \\
\geq 30 \%\end{array}$ & $\begin{array}{c}\Delta \text { pain score } \\
\geq 50 \%\end{array}$ \\
\hline Experimental & 2.0 & $37 \%$ & $59 \%$ & $65 \%$ & $35 \%$ \\
\hline group & 0.6 & $14 \%$ & $12 \%$ & $15 \%$ & $10 \%$ \\
\hline
\end{tabular}

Table 4. Average hypoalgesia across all sessions and all participants (left); proportion of hypoalgesia-inducing sessions for each group (right). 